\title{
OPEN Structural and microvascular changes of the peripapillary retinal nerve fiber layer in Von Hippel-Lindau disease: an OCT and OCT angiography study
}

\author{
Elisabetta Pilotto ${ }^{1}$, Elisabetta Beatrice Nacci ${ }^{2}{ }^{\bowtie}$, Gilda De Mojà ${ }^{1}$, \\ Alfonso Massimiliano Ferrara ${ }^{3}$, Raffaele Parrozzani ${ }^{1}$, Davide Londei ${ }^{1}$, Stefania Zovato $^{3}$ \& \\ Edoardo Midena ${ }^{1,2}$
}

Von Hippel-Lindau (VHL) disease is an autosomal dominant genetic disease caused by VHL gene mutation. Retinal hemangioblastomas $(\mathrm{RH})$ are vascularized tumors and represent the main ocular manifestation of the disease. Histopathologically, $\mathrm{RH}$ are composed of capillary vessels and stromal cells, the neoplastic population of the lesion. The origin of these stromal cells remains controversial, even if they are hypothesized to be glial cells. The aim of the present study was to investigate neuronal and microvascular changes of the peripapillary retinal nerve fiber layer, in which glial cells, neurons and capillaries (the radial peripapillary capillary plexus) interact. VHL patients with or without peripheral RH were enrolled and compared to healthy controls. Mean peripapillary retinal nerve fiber layer (pRNFL) thickness was measured by means of optical coherence tomography (OCT). The following vascular parameters of the radial peripapillary capillary plexus were quantified using OCT angiography: Vessel Area Density,Vessel Length Fraction, Vessel Diameter Index and Fractal Dimension. One hundred and nine eyes of 61 patients, and 56 eyes of 28 controls were consecutively studied. Mean pRNFL was significantly thinner in VHL eyes without RH versus eyes with RH and controls. Mean pRNFL thickness did not differ between VHL eyes with RH and controls. All OCTA vascular parameters were reduced in $\mathrm{VHL}$ eyes with or without $\mathrm{RH}$ versus controls, with significative difference for Vessel Diameter Index. The same OCTA parameters did not significantly differ between VHL eyes with or without RH. In VHL eyes without RH, pRNFL thinning may be the consequence of impaired perfusion of the radial peripapillary capillary plexus, while the increase of pRNFL thickness in VHL eyes with RH may depend on possible activation and proliferation of the other RNFL resident cells, the glial cells.

Heritable cancer syndrome Von Hippel-Lindau (VHL) is a genetic disease caused by VHL gene mutation ${ }^{1}$. VHL gene encodes a tumor suppressor protein (pVHL) which forms a VHL protein complex responsible for the degradation of hypoxia-inducible factors (HIFs). In absence of pVHL, HIFs increase with subsequent over-production of numerous growth factors, up-regulates angiogenesis and increases cellular proliferation. Pro-angiogenic and pro-mitotic stimuli lead to the development of various tumors including hemangioblastomas, typical vascularized hamartomatous lesions that develop in the central nervous system and in the retina ${ }^{2,3}$. Retinal hemangioblastoma $(\mathrm{RH})$ originate in the neurosensory retina or optic disc. They are histopathologically similar to central nervous system hemangioblastomas ${ }^{1}$ and composed of capillary vessels and stromal cells, the latter representing the neoplastic population ${ }^{2,4}$. The origin of these stromal cells remains controversial, even if they are hypothesized to be glial cells ${ }^{2,5-10}$. The advent of optical coherence tomography (OCT) and OCT angiography (OCTA) in clinical practice allows to explore single retinal layers and their vascularization, without any dye injection ${ }^{11}$. The aim of this research was to analyze, using OCT and OCTA, the structural and perfusion characteristics of

${ }^{1}$ Department of Ophthalmology, University of Padova, Padova University Hospital ERN-EYE Center, Padova, Italy. ${ }^{2}$ IRCCS, Fondazione G. B. Bietti, Via Livenza 3, 00198 Rome, Italy. ${ }^{3}$ Familial Tumor Unit, Veneto Institute of Oncology IOV-IRCCS, Padua, Padova, Italy. ${ }^{\bowtie}$ email: beatricenac@libero.it 


\begin{tabular}{|l|l|l|l|l|l|l|}
\hline $\begin{array}{l}\text { pRNFL thickness }(\boldsymbol{\mu m} \text { : } \\
\text { mean } \pm \text { SD) }\end{array}$ & VHL w/out RHs (71 eyes) & Healthy controls (56 eyes) & $\begin{array}{l}\text { VHL with RH } \\
\text { (38 eyes) }\end{array}$ & $\begin{array}{l}\text { VHL w/out RH } \\
\text { vs controls } \\
\boldsymbol{p} \text {-value }\end{array}$ & $\begin{array}{l}\text { VHL with RH vs VHL w/ } \\
\text { out RHs } \\
\boldsymbol{p} \text {-value }\end{array}$ & $\begin{array}{l}\text { VHL with RH vs } \\
\text { controls } \\
\boldsymbol{p} \text {-value }\end{array}$ \\
\hline Mean pRNFL & $100.7 \pm 13.4$ & $104.2 \pm 7.4$ & $103.5 \pm 9.9$ & $\mathbf{0 . 0 1 7 2}$ & $\mathbf{0 . 0 2 0 1}$ & 0.7421 \\
\hline T-pRNFL & $66.2 \pm 11.1$ & $71.7 \pm 14.2$ & $73.3 \pm 9.0$ & $\mathbf{0 . 0 4 5 4}$ & $\mathbf{0 . 0 0 0 2}$ & 0.5334 \\
\hline TS-pRNFL & $134.9 \pm 18.3$ & $144.6 \pm 16.3$ & $147.4 \pm 18.4$ & $\mathbf{0 . 0 1 2 7}$ & $\mathbf{0 . 0 0 0 5}$ & 0.6526 \\
\hline NS-pRNFL & $116.1 \pm 31.7$ & $112.4 \pm 19.7$ & $117.2 \pm 27.9$ & 0.6076 & 0.6338 & 0.4462 \\
\hline N-pRNFL & $78.5 \pm 18.8$ & $81.9 \pm 13.4$ & $76.3 \pm 15.6$ & 0.3735 & 0.7145 & 0.1471 \\
\hline NI-pRNFL & $119.3 \pm 26.1$ & $121.4 \pm 17.8$ & $111.2 \pm 14.5$ & 0.6727 & 0.0880 & $\mathbf{0 . 0 2 2 6}$ \\
\hline TI-pRNFL & $145.9 \pm 22.4$ & $150.9 \pm 19.0$ & $152.2 \pm 20.9$ & 0.2036 & $\mathbf{0 . 0 0 8 3}$ & 0.7188 \\
\hline PMB & $51.1 \pm 8.8$ & $52.9 \pm 7.4$ & $55.1 \pm 7.1$ & 0.3429 & $\mathbf{0 . 0 0 1 0}$ & $\mathbf{0 . 1 7 1 4}$ \\
\hline
\end{tabular}

Table 1. Peripapillary retinal nerve fiber layer (pRNFL) thickness in VHL eyes with or without RH and healthy controls. N-pRNFL, nasal sector-peripapillary Retinal Nerve Fiber Layer; NI-pRNFL, nasal-inferior sector-peripapillary Retinal Nerve Fiber Layer; NS-pRNFL, nasal-superior sector-peripapillary Retinal Nerve Fiber Layer; PMB, papillo-macular bundle; RH, retinal hemangioblastomas; T-pRNFL, temporal sectorperipapillary Retinal Nerve Fiber Layer; TI-pRNFL, temporal-inferior sector-peripapillary Retinal Nerve Fiber Layer; TS-pRNFL, temporal-superior sector-peripapillary Retinal Nerve Fiber Layer; VHL, Von Hippel Lindau disease; w/out, without. Significant p value in bold.

the peripapillary retinal nerve fiber layer, in which glial cells, neurons and capillaries (the radial peripapillary capillary plexus) interact, in VHL eyes with or without peripheral RH.

\section{Results \\ Population characteristics. From December 2018 to January 2020 sixty-one caucasian VHL patients and 28 heathy controls underwent enrolment. The groups were homogeneous for mean age $(39.0 \pm 13.8$ years vs $40.0 \pm 11.8, \mathrm{p}=0.7373$ ) and sex distribution (female/male: $32 / 28$ vs $17 / 11, \mathrm{p}=0.5591$ ). In 13 patients only one eye was evaluable, while fellow eye was excluded because of: retinal detachment (1 case), presence of $\mathrm{RH}$ at the posterior pole or optic disc ( 5 cases), band keratopathy ( 2 cases), epiretinal macular membrane ( 3 cases), macu- lar retinal pigment epithelium changes (1 case), myelinated nerve fibers ( 1 case). Therefore, 109 VHL eyes of 61 patients (38 eyes with $\mathrm{RH}[34.8 \%]$ and 71 without $\mathrm{RH}[65.1 \%]$ ), and 56 eyes of 28 healthy subjects were included.}

Peripapillary retinal nerve fiber layer thickness analysis. Mean Peripapillary Retinal Nerve Fiber Layer (pRNFL) thickness was significantly thinner in VHL eyes without RH compared to controls $(100.7 \pm 13.4 \mu \mathrm{m}$ vs $104.2 \pm 7.4 \mu \mathrm{m}, \mathrm{p}=0.0172)$. No difference was found between VHL eyes with RH and controls $(103.5 \pm 9.9 \mu \mathrm{m}$ vs $104.2 \pm 7.4 \mu \mathrm{m}, \mathrm{p}=0.7421)$ as pRNFL thickness is concerned. Mean pRNFL thickness was significantly thicker in VHL eyes with $\mathrm{RH}$ versus those without $\mathrm{RH}(103.5 \pm 9.9 \mu \mathrm{m}$ vs $100.7 \pm 13.4 \mu \mathrm{m}, \mathrm{p}=0.0201)$. In all temporal sectors, sectorial pRNFL was thicker in eyes with RH versus those without RH $(73.3 \pm 9 \mu \mathrm{m}$ vs $66.2 \pm 11.1 \mu \mathrm{m}$, $\mathrm{p}=0.0002$ for Temporal; $147.4 \pm 18.4 \mu \mathrm{m}$ vs $134.9 \pm 18.3 \mu \mathrm{m}, \mathrm{p}=0.0005$ for Temporal Superior; $152.2 \pm 20.9 \mu \mathrm{m}$ vs $145.9 \pm 22.4 \mu \mathrm{m}, \mathrm{p}=0.0083$ for Temporal Inferior; $55.1 \pm 7.1 \mu \mathrm{m}$ vs $51.1 \pm 7.1 \mu \mathrm{m}, \mathrm{p}=0.001$ for Papillo-Macular Bundle). Thinning of sectorial pRNFL in eyes without RH versus controls mostly involved the temporal sectors $(\mathrm{p}=0.0454$ for Temporal and $\mathrm{p}=0.0127$ for Temporal Superior). A detailed sectorial analysis of $\mathrm{pRNFL}$ thickness of all studied eyes is reported in Table 1 .

OCT-angiography vascular indexes analysis. All OCT-Angiography (OCTA) vascular parameters, including Vessel Area Density (VAD), Vessel Length Fraction (VLF), Vessel Diameter Index (VDI) and Fractal Dimension (FD), were reduced in VHL eyes with and without RH versus controls, reaching statistical significance for VDI ( $p=0.0365$ and $p=0.0293$, respectively). Figure 1 "Peripapillary OCT- Angiography capillary plexus in VHL eye (A) and Healthy control (B)".

OCTA vascular parameters did not significantly differ between VHL eyes with or without RH. (Table 2).

\section{Discussion}

In the present study, the peripapillary retinal nerve fiber layer was thinner in VHL eyes without RH, both compared to controls and to eyes with RH. At OCTA analysis of the radial peripapillary capillary plexus, the Vessel Diameter Index, which refers to the diameter of the vessels ${ }^{12}$, was significantly reduced in VHL eyes compared to healthy controls, independently of the presence or absence of $\mathrm{RH}$, showing a plexus characterized by analomalous thin capillaries. The radial peripapillary capillary plexus is a distinct retinal vascular network, located in the pRNFL, characterized by a specific radial distribution of capillaries, tightly coupling and suppling the nerve fibers ${ }^{11,13,14}$. Retinal blood flow is highly correlated to neural activity, in a strict neurovascular metabolic coupling $^{15}$. Therefore, the reduced perfusion of the this plexus may be correlated to the reduction in thickness of the RNFL, as occurs in other retinal diseases like diabetes and glaucoma, whose neurodegenerative nature is well demonstrated $^{13,16-18}$. In a retina-specific knockout VHL animal model, excessive vessel reduction and regression have already been found ${ }^{19}$. Therefore, an impaired radial peripapillary capillary plexus, characterized by reduction in peripapillary perfusion, may hypothetically cause reduction of retinal nerve fibers. 


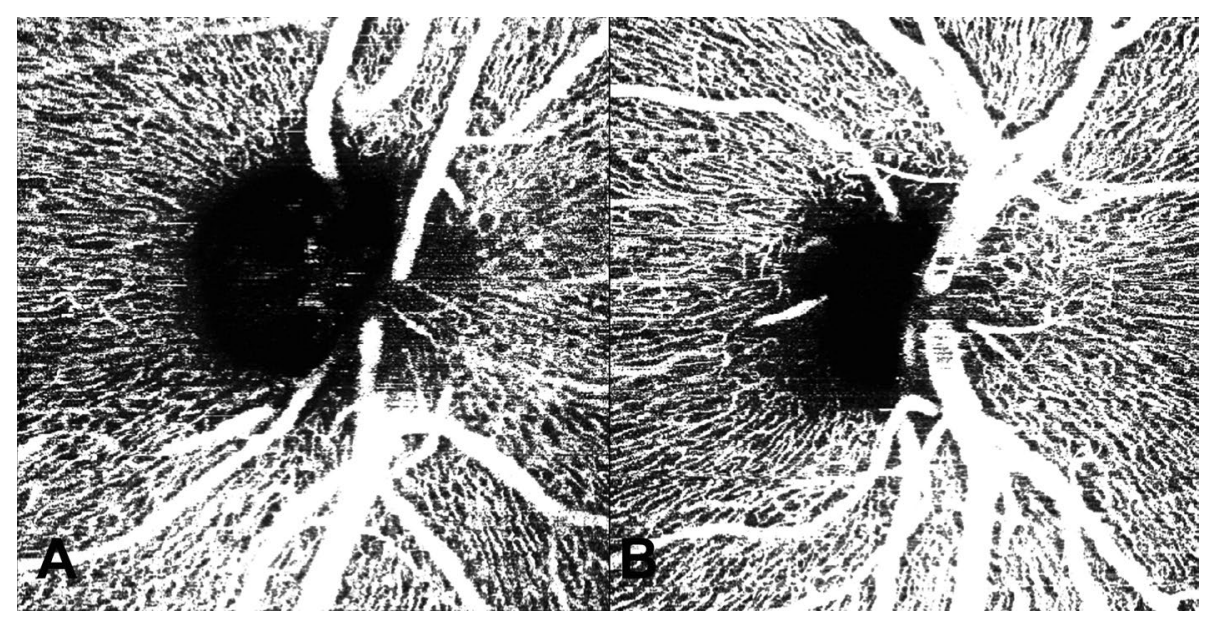

Figure 1. Peripapillary OCT-Angiography capillary plexus in VHL eye (A) and Healthy control (B). En face OCT-Angiography of the Radial Peripapillary Capillary Plexus showed reduced vascular perfusion in a VHL eye (A) compared to a healthy control (B). Quantification of plexus perfusion, expressed through evaluation of Vessel Area Density (VAD), Vessel Length Fraction (VLF), Vessel Diameter Index (VDI), Fractal Dimension on binary image (FDbin) and Fractal Dimension on skeletonized image (FDsk) indexes, detected a significant reduction in VHL eyes of VDI parameter.

\begin{tabular}{|c|c|c|c|c|c|c|}
\hline RPCP mean (SD) & VHL w/out RH (71eyes) & Healthy controls (56 eyes) & VHL with RH (38 eyes) & $\begin{array}{l}\text { VHL w/out RH vs } \\
\text { controls } \\
\text { p-value }\end{array}$ & $\begin{array}{l}\text { VHL with RH vs VHL w/ } \\
\text { out RHs } \\
\text { p-value }\end{array}$ & $\begin{array}{l}\text { VHL with RH } \\
\text { vs controls } \\
\text { p-value }\end{array}$ \\
\hline VAD & $0.4881(0.13)$ & $0.52390(0.09)$ & $0.5059(0.12)$ & 0.1248 & 0.4630 & 0.4609 \\
\hline VLF & $0.0853(0.02)$ & $0.0889(0.01)$ & $0.0885(0.01)$ & 0.2830 & 0.5038 & 0.9281 \\
\hline VDI & $5.6312(0.64)$ & $5.8827(0.53)$ & $5.6747(0.60)$ & 0.0293 & 0.7529 & 0.0365 \\
\hline FDbin & $1.8182(0.09)$ & $1.8466(0.04)$ & $1.8346(0.06)$ & 0.0929 & 0.6281 & 0.2860 \\
\hline FDsk & $1.5357(0.07)$ & $1.5568(0.04)$ & $1.5539(0.05)$ & 0.1628 & 0.4333 & 0.7518 \\
\hline
\end{tabular}

Table 2. OCT angiography parameters of the Radial Peripapillary Capillary Plexus (RPCP) in VHL eyes with or without RH and healthy controls. FDbin, Fractal Dimension of binary image; FDsk, Fractal Dimension of skeletonized images; RH, retinal hemangioblastomas; VAD, Vascular Area Density; VDI, Vessel Diameter Index; VHL:Von Hippel-Lindau Disease; VLF, Vessel Length Fraction. Significant p value in bold.

On the other hand, in VHL eyes with RH, showing the same radial peripapillary capillary plexus perfusion impairment, pRNFL was thicker than in eyes without RH. Therefore, radial peripapillary capillary plexus changes may be excluded in the pathophysiology of this relative thickening. The retinal nerve fiber layer is composed not only by nerve fibers and vessels, but also by glial cells, mostly Müller cells and astrocytes ${ }^{20,21}$ (from 18 to $42 \%$ ). Moreover, the thickness of pRNFL has been shown to be linearly correlated to the number of retinal astrocytes ${ }^{20}$. The role of astrocytes in VHL disease remains controversial, but histopathologic studies have already revealed trasformed and increased astrocytes in the stromal populations of hemangioblastomas, including the retinal ones $^{5,9,10,22-24}$. Under normal conditions, astrocytes maintain the homeostasis of the nervous tissue -controlling, protecting and supporting neuronal function- and regulate local blood flow ${ }^{25}$. Under pathological conditions, atrocytes react through a process called reactive astrogliosis, with hyperplasia/proliferation of the cells, increased number of their processes and larger cell body size $\mathrm{e}^{25,26}$. In an experimental model, the deletion of VHL gene in retinal atrocytes induced not only increased astrocyte cell number in the RNFL, but also upregulation of retinal VEGF production, which is a key factor in the pathophisiology and growth of hemangioblastomas ${ }^{27,28}$. Therefore, the relative increase in pRNFL thickness we detected in eyes with $\mathrm{RH}$ and not in eyes without $\mathrm{RH}$, represents in our view human in vivo clinical evidence of local activation of retinal astrocytes with consequent upregulation of retinal VEGF production, previoulsy demonstrated in animal models and in hystopathological studies.

In VHL patients, OCT and OCTA have been mainly used to study posterior pole RH or macular capillary changes related to $\mathrm{RH}^{29-32}$. To the best of our knowledge, this is the first OCT and OCTA study investigating the structural and perfusion characteristics of the peripapillary nerve fiber layer, in which glial cells, neurons and capillaries of the radial peripapillary capillary plexus interact.

Genotype-phenotype correlations were not investigated in the present research. This could represent a limit of the study, since we cannot exclude that the genotype of VHL germline mutation may differently influence the analyzed parameters, as higher prevalence of progression-related complications and preretinal fibrosis have already been correlated to $\mathrm{it}^{33,34}$. A further analysis of genotype versus OCT and OCTA parameters is ongoing. 
In conclusion, in VHL eyes, pRNFL is thinner than in controls hypothetically as a consequence of reduced perfusion of the radial peripapillary capillary plexus, as shown in other retinal disorders. When peripheral $\mathrm{RH}$ are present, pRNFL thickness relatively increases compared to eyes without RH. This fact may be due to the proliferation of retinal astrocytes, as previously histologically reported. This observation may offer a holistic interpretation of the involvement of different retinal cells in VHL progressive ocular disease. A clinical application of our findings may be that, in the follow-up of VHL patients, the increase of pRNFL thickness, compared to baseline, may guide retinal examination for peripheral RH screening. Larger population and longer follow up are mandatory to confirm our observations.

\section{Methods}

This was a cross-sectional study and informed consent was obtained from each subject; data collection was compliant with the tenets of the Declaration of Helsinki. The approval from the Ethics Committee for Clinical Practice of the Azienda Ospedaliera di Padova for the study was obtained (Prot.34971/AOP/2018). VHL patients, referred by the Familial Tumor Unit of the Veneto Institute of Oncology (IOV-IRCCS), underwent scheduled eye examination including: best-corrected visual acuity (BCVA) measurement using standard Early Treatment Diabetic Retinopathy Study (ETDRS) charts, anterior segment evaluation at slit lamp, intraocular pression measurement, fundus examination by means of indirect ophthalmoscopy and 90-diopter-lens biomicroscopy. Patients who presented congenital or acquired anterior segment diseases; epiretinal macular membrane; posterior pole and optic nerve - treated or untreated RH; glaucoma; refractive errors $\geq 6$ diopters; history of ocular inflammation; concomitant presence of other retinal vasculopathies were not included in the research. The posterior pole and optic nerve $\mathrm{RH}$ were excluded because they may interfere with the correct visualization and quantification of RNFL and vascular parameters. Moreover, patients with pituitary stalk hemangioblastomas, which may compromise optic nerve pathway, were also excluded ${ }^{35}$. An age-matched healthy group was recruited for comparison.

OCT and OCT angiography. After pupil dilatation (obtained with 1\% tropicamide eye-drops solution), enrolled eyes performed OCT and OCTA using Spectralis HRA + OCTA (Heidelberg Engineering, Heidelberg, Germany) in a semi-dark room, late in the morning and with the in-built eye-tracker always active to obtain high quality scans and correct foveal centration of the scans. The OCT scan protocol included a peripapillary $3.5 \mathrm{~mm}$ ring N-RNFL (N-site/axonal menu) scan, centered onto the optic nerve head, to automatically provide the peripapillary retinal nerve fiber layer ( $\mathrm{pRNFL}$ ) thickness value. pRNFL thickness was expressed both as mean pRNFL (pRNFL) and as sectorial pRNFL (Temporal, T-pRNFL; Temporal Superior, TS-pRNFL; Nasal Superior, NS-pRNFL; Nasal, N-pRNFL; Nasal Inferior, NI-pRNFL; Temporal Inferior, TI-pRNFL; Papillo-Macular Bundle, PMB).

An OCTA scan pattern of $10^{\circ} \times 10^{\circ}(3.0 \times 3.0 \mathrm{~mm} ; 512 \mathrm{~B}$-scans separated by 6 micron $)$ centered onto the optic nerve head was acquired. The inbuilt software automatically generated the enface OCTA image of the radial peripapillary capillary plexus, which is the radial capillary monolayer network encircling the optic disc ${ }^{11,13}$. As previously described ${ }^{29}$ in order to guarantee high quality OCTA images, only images with a signal strength more than 30 in "Q score" (on a scale of 0 to 40 for Spectralis, Heidelbeerg), were analysed ${ }^{36,37}$. Correct foveal centration of the scans was obtained with the inbuilt eye tracking system. A skilled technician checked each image after acquisition to detect any motion artifacts or segmentation errors, and eventually repeated examination. Open-source available ImageJ software (National Institutes of Health, Bethesda, MD, USA) allowed quantitative analysis of the OCTA en face images, obtaining quantitative parameters of the radial peripapillary capillary plexus: Vessel Area Density (VAD), Vessel Length Fraction (VLF), Vessel Diameter Index (VDI) and Fractal Dimension (FD), as previously described ${ }^{12,38}$. OCTA en face images were automatically converted into a binary image. VAD, which provides the estimate of real vessel density in the examined area, taking into consideration both vessel length and vessel diameter, was obtained dividing the number of black pixels in the binary image by the total number of image pixels. The binarized image was then "skeletonized" deleting the outer boundary of the binarized image so that each vessel segment had a single pixel width. By dividing the number of vessel pixels in the skeletonized image by the total number of image pixels VLF was obtained, quantifing the vessel length regardless of the vessel diameters. VDI parameter, sensitive to vascular dilation in the OCT-A images as it presents the vessel size information regardless of the vessel length, was obtained by processing both the binary and the skeletonized images to calculate the average vessel caliber. FD, representing complexity of images, was calculated both over the skeletonized images (FDsk) using a box counting technique and over binary images (FDbin) ${ }^{12,38}$.

Statistical analysis. All variables were summarized according to the conventional methods of descriptive statistics: mean and standard deviation for quantitative variables; absolute and relative (percentage) frequencies for qualitative variables. Normal distribution of parameters was checked by Shapiro-Wilk's test.

Gender distribution and age of enrolled patients and controls were compared using chi-square test and $\mathrm{t}$-Student test for independent samples, respectively. Mean pRNFL and sectorial pRNFL thickness values were analyzed with one-way ANOVA model. When applicable, statistical models were adjusted for replication of measures in both eyes of patients and controls.

A mixed effects ANOVA model was applied to compare OCTA parameters between groups, with repeated measures (both eyes), and adjusted for age. Statistical significance was set at $\mathrm{p}<0.05$ level. All the analyses were performed by SAS ${ }^{\circledast} 9.4$ statistical software (SAS Institute, Cary, NC, USA).

\section{Data availability}

The authors have full access to all the data and take responsibility for the integrity of the data and the accuracy of the data analysis as well as the decision to submit to publication. 
Received: 30 September 2020; Accepted: 10 December 2020

Published online: 08 January 2021

\section{References}

1. Aronow, M. E. et al. Von Hippel lindau disease: update on pathogenesis and systemic aspects. Retina 39, 2243-2253 (2019).

2. Pierscianek, D. et al. Study of angiogenic signaling pathways in hemangioblastoma. Neuropathology. 37, 3-11 (2017).

3. Karimi, S., Arabi, A., Shahraki, T. \& Safi, S. V. Hyppel-Lindau disease and the eye. J. Ophthalmic. Vis. Res. 15, 78-94 (2020).

4. Ishizawa, K., Komori, T. \& Hirose, T. Stromal cells in hemangioblastoma: neuroectodermal differentiation and morphological similarities to ependymoma. Pathol. Int. 55, 377-385 (2005).

5. Grossniklaus, H. E., Thomas, J. W., Vignesvaran, N. \& Jarrett, W. H. 3rd. Retinal hemangioblastoma. A histologic, immunohistochemical, and ultrastructural evaluation. Ophthalmology 99, 140-145 (1992).

6. Vortmeyer, A. O. \& Alomari, A. K. Pathology of the nervous system in von Hippel-Lindau Disease. J. Kidney Cancer VHL 2, 114-129 (2015).

7. Shively, S. B. et al. Developmentally arrested structures preceding cerebellar tumors in von Hippel-Lindau disease. Mod. Pathol. 24, 1023-1030 (2011).

8. Park, D. M. et al. von Hippel-Lindau disease-associated hemangioblastomas are derived from embryologic multipotent cells. PLoS Med. 4, 60 (2007).

9. Takada, S., Hojo, M., Takebe, N., Tanigaki, K. \& Miyamoto, S. Stromal cells of hemangioblastomas exhibit mesenchymal stem cell-derived vascular progenitor cell properties. Brain Tumor. Pathol. 35, 193-201 (2018).

10. Nicholson, D. H., Green, W. R. \& Kenyon, K. R. Light and electron microscopic study of early lesions in angiomatosis retinae. Am. J. Ophthalmol. 82, 193-204 (1976).

11. Campbell, J. P. et al. Detailed vascular anatomy of the human retina by projection-resolved optical coherence tomography angiography. Sci. Rep. 7, 42201 (2017).

12. Kim, A. Y. et al. Quantifying microvascular density and morphology in diabetic retinopathy using spectral-domain optical coherence tomography angiography. Invest Ophthalmol Vis Sci. 57, 362-370 (2016).

13. Spaide, R. F. Measurable aspects of the retinal neurovascular unit in diabetes, glaucoma, and controls. Am. J. Ophthalmol. 207, 395-409 (2019).

14. Parrozzani, R. et al. Retinal vascular abnormalities in a large cohort of patients affected by neurofibromatosis type 1: A study using optical coherence tomography angiography. Retina 38, 585-593 (2018).

15. Prada, D. et al. Autoregulation and neurovascular coupling in the optic nerve head. Surv. Ophthalmol. 61, 164-186 (2016).

16. Midena, E. \& Pilotto, E. Emerging insights into pathogenesis. Dev. Ophthalmol. 60, 16-27 (2017).

17. Frizziero, L., Parrozzani, R., Londei, D., Pilotto, E., Midena, E. Quantification of vascular and neuronal changes in the peripapillary retinal area secondary to diabetic retinopathy. Br. J. Ophthalmol. (2020) (in press).

18. Rodrigues, T. M. et al. Peripapillary neurovascular coupling in the early stages of diabetic retinopathy. Retina 39, 2292-2302 (2019).

19. Kurihara, T. et al. von Hippel-Lindau protein regulates transition from fetal to adult circulatory system in retina. Development 137, 1563-1571 (2010)

20. Büssow, $\mathrm{H}$. The astrocytes in the retina and optic nerve head of mammals: a special glia for the ganglion cell axons. Cell Tissue Res. 206, 367-378 (1980).

21. Ogden, T. E. Nerve fiber layer of the primate retina: thickness and glial content. Vision Res. 23, 581-587 (1983).

22. Vortmeyer, A. O. et al. Morphologic and genetic analysis of retinal angioma associated with massive gliosis in a patient with von Hippel-Lindau disease. Graefes Arch. Clin. Exp. Ophthalmol. 237, 513-517 (1999)

23. Jakobiec, F. A., Font, R. L. \& Johnson, F. B. Angiomatosis retinae. An ultrastrtuctural study and lipid analysis. Cancer 38, 2042-2056 (1976).

24. Hasselblatt, M. et al. Cellular and reticular variants of haemangioblastoma revisited: a clinicopathologic study of 88 cases. Neuropathol. Appl. Neurobiol. 31, 618-622 (2005).

25. De Hoz, R et al. Retinal macroglial responses in health and disease. Biomed. Res. Int. 2954721 (2016).

26. Acaz-Fonseca, E., Ortiz-Rodriguez, A., Azcoitia, I., Garcia-Segura, L. M. \& Arevalo, M. A. Notch signaling in astrocytes mediates their morphological response to an inflammatory challenge. Cell Death Discov. 5, 85-103 (2019).

27. Weidemann, A. et al. Astrocyte hypoxic response is essential for pathological but not developmental angiogenesis of the retina. Glia 58, 1177-1185 (2020).

28. Kaelin, W. G. Jr. Molecular basis of the VHL hereditary cancer syndrome. Nat. Rev. Cancer 2, 673-682 (2002).

29. Pilotto, E. et al. Macular Perfusion impairment in Von Hippel-Lindau disease suggests a generalized retinal vessel alteration. $J$ Clin. Med. 9, 2677 (2020).

30. Sagar, P., Rajesh, R., Shanmugam, M., Konana, V. K. \& Mishra, D. Comparison of optical coherence tomography angiography and fundus fluorescein angiography features of retinal capillary hemangioblastoma. Indian J. Ophthalmol. 66, 872-876 (2018).

31. Lang, S. J. et al. Value of optical coherence tomography angiography imaging in diagnosis and treatment of hemangioblastomas in von Hippel-Lindau disease. Ophthal. Surg. Lasers Imaging Retina 47, 935-946 (2016).

32. Lu, Y. et al. Detection of retinal microvascular changes in von Hippel-Lindau disease using optical coherence tomography angiography. PloSone 15, e0229213 (2020).

33. Hajjaj, A. et al. Retinal haemangioblastomas in von Hippel-Lindau germline mutation carriers: progression, complications and treatment outcome. Acta Ophthalmol. https://doi.org/10.1111/aos.14360 (2020).

34. Dollfus, H. et al. Retinal hemangioblastomas in von Hippel-Lindau disease: a clinical and molecular study. Invest. Ophthalmol. Vis. Sci. 43, 3067-3074 (2002).

35. Lonser, R. R., Butman, J. A., Kiringoda, R., Song, D. \& Oldfield, E. H. Pituitary stalk hemangioblastomas in von Hippel Lindau disease. J. Neurosurg. 110, 350-353 (2009).

36. Lim, H. B., Kim, Y. W., Kim, J. M., Jo, Y. J. \& Kim, J. Y. The importance of signal strenght in quantitative assessment of retinal vessel density using optical coherence tomography angiography. Sci. Rep. 8, 12897 (2018).

37. Huang, Y. et al. Signal quality assessment of retinal optical coherence tomography images. Invest. Ophthalmol. Vis. Sci. 53, 21332141 (2012).

38. Parrozzani, R. et al. Peripapillary vascular changes in radiation optic neuropathy: an optical coherence tomography angiography grading. Br. J. Ophthalmol. 102, 1238-1243 (2018).

\section{Acknowledgements}

There are no competing interests, financial and non-financial, for any author. The research contribution by the G.B. Bietti Foundation was supported by Fondazione Roma and Ministry of Health. The authors thank Fabiano Cavarzeran, Department of Ophthalmology, University of Padova, for the statistical elaboration of the data. The authors are grateful to the European Reference Network dedicated to Rare Eye Diseases (ERN-EYE) for useful discussions. 


\section{Author contributions}

E.P., E.B.N., E.M.: study conception, design, interpretation of data, drafting and revising; final approval and agreement to be accountable for all aspects of the work. G.D.M., A.M.F., R.P., D.L., S.Z.: data acquisition/analysis/interpretation, revising of work, final approval and agreement to be accountable for all aspects of the work.

\section{Funding}

This research received no specific grant from any funding agency in the public, commercial or not-for-profit sectors.

\section{Competing interests}

The authors declare no competing interests.

\section{Additional information}

Correspondence and requests for materials should be addressed to E.B.N.

Reprints and permissions information is available at www.nature.com/reprints.

Publisher's note Springer Nature remains neutral with regard to jurisdictional claims in published maps and institutional affiliations.

(c) (i) Open Access This article is licensed under a Creative Commons Attribution 4.0 International License, which permits use, sharing, adaptation, distribution and reproduction in any medium or format, as long as you give appropriate credit to the original author(s) and the source, provide a link to the Creative Commons licence, and indicate if changes were made. The images or other third party material in this article are included in the article's Creative Commons licence, unless indicated otherwise in a credit line to the material. If material is not included in the article's Creative Commons licence and your intended use is not permitted by statutory regulation or exceeds the permitted use, you will need to obtain permission directly from the copyright holder. To view a copy of this licence, visit http://creativecommons.org/licenses/by/4.0/.

(C) The Author(s) 2021 